\title{
Pharmacotherapy of kidney transplant rejection: Current therapy and future aspects
}

\author{
Naim Kittana ${ }^{1}$, Alaa Hamamri ${ }^{1}$, Lana Zighan ${ }^{1}$, ISRAA SALAMA ${ }^{1}$, and Suhaib Hattab ${ }^{1}$ \\ ${ }^{1}$ An-najah National University Faculty of Medicine and Health Sciences
}

May 12, 2020

\begin{abstract}
Renal transplantation is the ultimate therapeutic option for end-stage renal failure. A major challenge that often leads to the loss of the renal graft is the immune-mediated organ rejection, of which there are three types; hyperacute, acute and chronic rejection. This necessitates a proper use of immunosuppressants in the form of short-term induction therapy, as prophylaxis against early acute rejection, and a life-long maintenance therapy against chronic rejection. However, the suppression of the immune system would also compromise the ability of the immune system to eradicate the pathogens and early cancer cells, which increases the risk for various types of serious infections and malignancies. Some of these drugs are also associated with hematologic, metabolic, cardiovascular and renal toxicities. This manuscript thoroughly reviews the available clinical data on the currently approved drugs that are used for both the induction and maintenance therapy, with some focus on their pharmacology. It also discusses the current guidelines and covers, in some details, the new drug classes that are still under clinical investigations.
\end{abstract}

\section{Hosted file}

kidney-transplantation-11-05-2020 Final version.docx available at https://authorea.com/users/ 320958/articles/450586-pharmacotherapy-of-kidney-transplant-rejection-current-therapyand-future-aspects 